\title{
Comparison of Three Segmentation Methods for Breast Ultrasound Images Based on Level Set and Morphological Operations
}

Dewi Putrie Lestari, Sarifuddin Madenda, Ernastuti, Eri Prasetyo Wibowo

Department of Computer Science and Information Technology, Gunadarma University, Indonesia

\begin{tabular}{l}
\hline Article Info \\
\hline Article history: \\
Received Jun 5, 2016 \\
Revised Nov 25, 2016 \\
Accepted Dec 15, 2016
\end{tabular}

Keyword:

Breast lesion

Level set

Morphological operations

Segmentation

Ultrasound

\begin{abstract}
Breast cancer is one of the major causes of death among women all over the world. The most frequently used diagnosis tool to detect breast cancer is ultrasound. However, to segment the breast ultrasound images is a difficult thing. Some studies show that the active contour models have been proved to be the most successful methods for medical image segmentation. The level set method is a class of curve evolution methods based on the geometric active contour model. Morphological operation describes a range of image processing technique that deal with the shape of features in an image. Morphological operations are applied to remove imperfections that introduced during segmentation. In this paper, we have evaluated three level set methods that combined with morphological operations to segment the breast lesions. The level set methods that used in our research are the Chan Vese (CV) model, the Selective Binary and Gaussian Filtering Regularized Level Set (SBGFRLS) model and the Distance Regularized Level Set Evolution (DRLSE) model. Furthermore, to evaluate the method, we compared the segmented breast lesion that obtained by each method with the lesion that obtained manually by radiologists. The evaluation is done by four metrics: Dice Similarity Coefficient (DSC), True-Positive Ratio (TPR), TrueNegative Ratio (TNR), and Accuracy (ACC). Our experimental results with 30 breast ultrasound images showed that the $\mathrm{C}-\mathrm{V}$ model that combined with morphological operations have better performance than the other two methods according to mean value of DSC metrics.
\end{abstract}

Copyright $\odot 2017$ Institute of Advanced Engineering and Science. All rights reserved.

\section{Corresponding Author:}

Dewi Putrie Lestari,

Department of Computer Science and Information Technology,

Gunadarma University,

Margonda Raya Road, Pondok Cina, Depok, West Java 16424, Indonesia.

Email: dewiputrie26@gmail.com

\section{INTRODUCTION}

Breast cancer is one of the leading causes of death among women both in developed countries and in developing countries [1]. The early detection can reduce the death rate and helpful the treatment of breast cancer [2]. However, early detection requires an accurate and reliable diagnosis that be able to distinguish benign and malignant lesions, where imaging techniques play an important task [3]. One of the most frequently used diagnosis tools to detect breast cancer is ultrasound [4].

Ultrasound image segmentation is a difficult task because these images contain strong speckle noise and attenuation artifacts [5]. Several studies show that the active contour models have been proved to be the most successful methods for image segmentation [6]. The basic idea of the active contour model is to develop a curves under some constraints to extract the desired object [7]. There are two types of active contour models: parametric active contour models and geometric active contour models [8]. The difference of 
parametric active contour models and geometric active contour models is that the former represent curves and surfaces explicitly in their parametric forms, but the latter represent curves and surfaces implicitly [9].

Geometric active contour models have many advantages over parametric active contour models, such as computational simplicity and the ability to change curve topology during deformation [9]. The level set method is a class of curve evolution method based on the geometric active contour models, which this method introduced by Osher-Sethian that overcame the problems of the associated with classical energy minimization approaches [9]. The basic idea of the level set method is to represent a contour as the zero level set of a higher dimensional function, called a level set function (LSF), and formulate the motion of the contour as the evolution of the level set function [10]. A desirable advantage of the level set method is that they can represent contours of complex topology and are able to handle topological changes, such as splitting and merging, in a natural and efficient way, which is not allowed in parametric active contour models [10].

In this paper, we compared three level set methods that combined with morphological operations for automatic segmentation of breast ultrasound images. Three level set models used in this paper are the Chan Vese (C-V) model [11], the Selective Binary and Gaussian Filtering Regularized Level Set (SBGFRLS) model [7] and the Distance Regularized Level Set Evolution (DRLSE) model [10]. Furthermore, to evaluated the methods, we compared the segmented breast lesion that obtained by each method with the lesion that obtained manually by radiologists using area-based segmentation assessment metrics.

\section{RESEARCH METHOD}

Tumor area on breast ultrasound images obtained by means perform the segmentation process. Intake of the tumor area should be done properly, because the contour of segmentation result is very establish the truth of the grouping process the types of tumors. Therefore, we need to measure the accuracy of the segmentation methods that used in this research. This is done by performing validation of the segmentation method proposed or Region of Interest (ROI) images against the segmentation manually by an experienced radiologist or Ground Truth (GT) image. The research stages in this paper are shown by a research scheme in Figure 1.

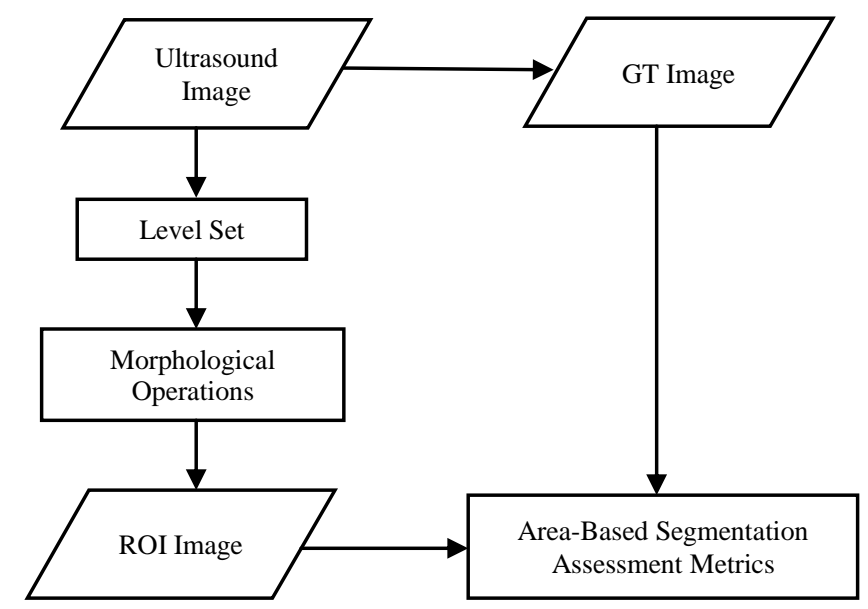

Figure 1. Scheme of the Research Stages

Based on Figure 1, we can conclude that this research is divided into two stages. The first stage is the segmentation of the breast ultrasound image and the second stage is validation the results of segmentation method. The first stage begins with a segmentation process of breast ultrasound images using level set algorithm. The next process is the application of morphological operations to the image of the results of level set method. This segmentation process will produce the Region of Interest (ROI) image. Then in the ultrasound images also done segmentation manually that produced the Ground Truth (GT) image. The validation process is done by comparing the ROI image with the GT image using area-based segmentation assessment metrics method.

\subsection{Segmentation Methods}

Image segmentation is a process of dividing the image into several areas homogeneous based on certain similarity criteria such as intensity, color and texture [12]. The automatic segmentation process is a 
necessary step in analysis of medical images. However, the accurate and reliable segmentation methods are a key requirement for the extraction of qualitative or quantitative information from images [13]. In this research, to segment the breast lesion we used three methods based on level set that each method combined with morphological operations. Three level set models used in this paper are the Chan Vese model, the Selective Binary and Gaussian Filtering Regularized Level Set model and the Distance Regularized Level Set Evolution model.

\subsubsection{Chan Vese Model}

Chan and Vese proposed a region-based active contour models which is a special case of MumfordShah formulation to handle problems of edge-based active contour models [1]. Let $\Omega$ is a domain for a given image $I(x, y)$, they represented a contour $C$ implicitly through Lipschitz function $\phi(x, y): \Omega \rightarrow \mathfrak{R}$ that defined as follows:

$$
\left\{\begin{array}{l}
C=\{(x, y) \in \Omega: \phi(x, y)=0\} \\
\text { insid }(C)=\{(x, y) \in \Omega: \phi(x, y)>0\} \\
\text { outsid }(C)=\{(x, y) \in \Omega: \phi(x, y)<0\}
\end{array}\right.
$$

Segmentation using Chan Vese (C-V) model is done by minimizing an energy function [7]. The formula function this energy expressed by using Heaviside function that defined in Equation 2 and Dirac function that defined in Equation 3.

$$
\begin{aligned}
& H(\phi)= \begin{cases}1, & \text { jika } \phi \geq 0 \\
0, & \text { jika } \phi<0\end{cases} \\
& \delta(\phi)=\frac{d}{d \phi} H(\phi)
\end{aligned}
$$

Furthermore, Chan and Vese formulate energy function that shown by the formula [9]:

$$
\begin{aligned}
& \left.F\left(c_{1}, c_{2}, \phi\right)=\mu \int_{\Omega} \delta(\phi(x, y)) \mid \nabla \phi(x, y)\right) d x d y+v \int_{\Omega} H(\phi(x, y)) d x d y \\
& +\lambda_{1} \int_{\Omega}\left|I(x, y)-c_{1}\right|^{2} H(\phi(x, y)) d x d y+\lambda_{2} \int_{\Omega}\left|I(x, y)-c_{2}\right|^{2}(1-H(\phi(x, y))) d x d y
\end{aligned}
$$

where $\mu \geq 0, v \geq 0, \lambda_{1} \geq 0, \lambda_{2} \geq 0$, are fixed parameters, $\mu$ controls the smoothness of zero level set, $v$ increases the propagation speed, $\lambda_{1}$ control the image data driven force inside, $\lambda_{2}$ control the image data driven force outside the contour, $\nabla$ is the gradient operator, $c_{1}$ is is the average intensities inside the contour that represented in Equation (5), and $c_{2}$ is the average intensities outside the contour that represented in Equation 6 [7].

$$
\begin{gathered}
c_{1}(\phi)=\frac{\int_{\Omega} I(x, y) H(\phi(x, y)) d x d y}{\int_{\Omega} H(\phi(x, y)) d x d y} \\
c_{2}(\phi)=\frac{\int_{\Omega} I(x, y)(1-H(\phi(x, y))) d x d y}{\int_{\Omega}(1-H(\phi(x, y))) d x d y}
\end{gathered}
$$

By incorporating the length and area energy terms into Equation 4 and minimizing them, the corresponding variational level set formulation is as follows [7]:

$$
\frac{\partial \phi}{\partial t}=\delta(\phi)\left[\mu \nabla\left(\frac{\nabla \phi}{|\nabla \phi|}\right)-v-\lambda_{1}\left(I(x, y)-c_{1}\right)^{2}+\lambda_{2}\left(I(x, y)-c_{2}\right)^{2}\right]
$$




\subsubsection{Selective Binary and Gaussian Filtering Regularized Level Set Model}

Selective Binary and Gaussian Filtering Regularized Level Set (SBGFRLS) model utilizes the advantages of both edge-based active contour models and region-based active contour models [14]. Edge-based models use image gradients to construct an Edge Stopping Function (ESF) to stop the contour evolution on the object boundaries [7]. In SBGFRLS model has been developed a region based Singed Pressure Force (SPF) function to substitute the ESF, which is able to control the direction of evolution [7]. The SPF function has opposite signs (range of SPF function is [-1 1]) around the boundaries of the object, so the contour can expand when it is inside the boundary and can shrinks when it is outside the boundary [7]. The SPF function in this model is defined by the formula [14]:

$$
\operatorname{spf}(I(x, y))=\frac{I(x, y)-\frac{c_{1}+c_{2}}{2}}{\max \left(\left|I(x, y)-\frac{c_{1}+c_{2}}{2}\right|\right)}
$$

with $c_{1}$ and $c_{2}$ are expressed in Equation 5 and Equation 6, respectively.

Then by substituting SPF function into the Equation 5, we obtained the level set formulation that defined as [14]:

$$
\frac{\partial \phi}{\partial t}=\operatorname{spf}(I(x, y))\left[\operatorname{div}\left(\frac{\nabla \phi}{|\nabla \phi|}\right)+\alpha\right]|\nabla \phi|+\nabla \operatorname{spf}(I(x, y)) \cdot \nabla \phi
$$

The regular term div $\left(\frac{\nabla \phi}{|\nabla \phi|}\right)|\phi|$ is unnecessary because Gaussian filter can be utilized to smooth the level set function to keep the interface regular. Beside that, the term $\nabla \operatorname{spf} \cdot \nabla \phi$ can be removed because the method utilizes the statistical information of the regions. Thus the final level set model is given by the formula [14]:

$$
\frac{\partial \phi}{\partial t}=\operatorname{spf}\left(u_{0}(x, y)\right) \cdot \alpha|\nabla \phi|
$$

\subsubsection{Distance Regularized Level Set Evolution Model}

The problem of traditional level set method is high computational cost which caused use reinitialization. $\mathrm{Li}$ et al. proposed a level set method without reinitialization by integrating a penalty term into the energy functional [2]. More recently, by adding a distance regularization term into energy functional, Li et al. presented a novel distance regularized level set evolution method, that is, Distance Regularized Level Set Evolution (DRLSE) model [2]. This model can not only completely eliminate the need for reinitialization but also avoid the undesirable side effect [2].

Let $\Omega \subset R^{2}$ be a bounded Lipschitz image domain. The energy functional $E(\cdot)$ is defined by the following function [2]:

$$
E(\phi)=\mu R_{p}(\phi)+E_{e x t}(\phi)
$$

with $R_{p}(\phi)$ is the distance regularization term defined in Equation (12) and $E_{\text {ext }}(\phi)$ is the external energy is written in Equation (13) [2].

$$
\begin{aligned}
& R_{p}(\phi)=\int_{\Omega} p(|\phi|) d x \\
& E_{\text {ext }}(\phi)=\lambda \int_{\Omega} g \delta(\phi)|\nabla \phi| d x+\alpha \int_{\Omega} g H(\phi) d x
\end{aligned}
$$

$p$ is the potential function for $R_{p}(\phi)$ that defined in Equation 14 and $g$ is an ESF such that $\lim _{t \rightarrow \infty} g(t)=0$, that defined in Equation 15 [2]. 


$$
\begin{aligned}
& p(s)=\left\{\begin{array}{l}
\frac{1}{(2 \pi)^{2}}(1-\cos (2 \pi s)), \quad s \leq 1 \\
\frac{1}{2}(s-1)^{2}, \quad s>1
\end{array}\right. \\
& g=\frac{1}{1+\left|\nabla G_{\sigma} * I\right|^{2}}
\end{aligned}
$$

where $G_{\sigma}$ is a Gaussian function with the standard deviation $\sigma, I$ is a given 2D image, symbol $*$ represent convolution, and $|\cdot|$ is the modulus of the smoothed image gradients [2].

The associated Euler-Lagrange equation, we obtained by minimizing function Equation 10 with respect to $\phi$ is defined as follows [2]:

$$
\frac{\partial \phi}{\partial t}=\mu \operatorname{div}\left(d_{p}(|\nabla \phi|) \nabla \phi\right)+\lambda \delta(\phi) \operatorname{di}\left(g \frac{\nabla \phi}{|\nabla \phi|}\right)+\alpha g \delta_{\varepsilon}(\phi)
$$

where $\operatorname{div}(\cdot)$ is the divergence operator and $d_{p}$ is a function given by the formula [2]:

$$
d_{p}(s)=\frac{p^{\prime}(s)}{s}
$$

\subsubsection{Morphological Operations}

Morphological operation describes a range of image processing technique that deal with the shape of features in an image [4]. Morphological operations are applied to remove imperfections that introduced during segmentation. Two basic morphological operations are dilation and erosion. They are defined in terms of more elementary set operations, but are employed as the basic elements of many algorithms [15].

Dilation and erosion are produced by the interaction of a set called a structuring element (SE) with a set of pixels of interest in the image [15]. Let $A(x, y)$ be a set of pixels and let $B(s, t)$ a SE, then the morphological dilation of image $A$ by the structuring element $B$ is defined in Equation (18) and the morphological erosion of image $A$ by the structuring element $B$ is defined in Equation (19). The morphological dilation will expand the components of an image but and the morphological erosion will shrink them [4].

$$
\begin{aligned}
& A(x, y) \oplus B(s, t)=\max \{A(x-s, y-t)+B(s, t)\} \\
& A(x, y) \Theta B(s, t)=\min \{A(x+s, y+t)-B(s, t)\}
\end{aligned}
$$

Furthermore, dilation and erosion operations can be combined with each other. The combination of morphological operations that often used are opening and closing. The opening operations of image $A$ by SE $B$, is denoted $A \circ B$, is defined in Equation 20. Which says the opening of image $A$ by SE $B$ is the erosion of image $A$ by $\operatorname{SE} B$, followed by a dilation of the result by $\operatorname{SE~} B$ [12]. The opening operations generally smoothes the contour of an object, breaks narrow isthmutes, and eliminates thin protrusions [4].

The closing operations of image $A$ by $\operatorname{SE} B$, denoted $A \bullet B$, is defined in Equation 21. Which says the closing of image $A$ by SE $B$ is simply the dilation of image $A$ by $\operatorname{SE} B$, followed by the erosion of the result by SE $B$ [12]. The closing operations also tends to smooth sections of contours but, as opposed to opening, it generally fuses narrow breaks and long thin gulfs, eliminates small holes, and fills gaps in the contour [4].

$$
\begin{aligned}
& A \circ B=(A \Theta B) \oplus B \\
& A \bullet B=(A \oplus B) \Theta B
\end{aligned}
$$

\subsection{Validation Methods}

The proposed methods in this paper have been evaluated using area-based segmentation assessment metrics. Area-based segmentation assessment metrics measure the amount of area overlap between the 
obtained segmentation and the Ground Truth. In the illustrated Figure 2, let $A$ represented the segmented region to be assessed and $M$ describe the manually delineated or GT reference region to be assessed, it will form 4 regions: True-Positive (TP), True Negative (TN), False-Positive (FP), and False Negative (FN). The $\mathrm{TP}$ area corresponds to the correctly segmented areas belonging to the lesion, the TN area corresponds to the correctly segmented areas belonging to the background of the image, the FP area corresponds to the areas wrongly labeled as a lesion since this areas don't belong to the reference delineation, and the FN area corresponds to the areas of the true segmentation that have been missed by the segmentation under assessment [4].

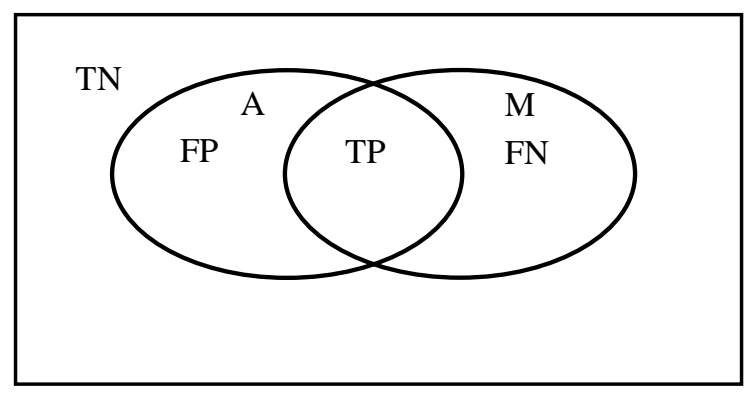

Figure 2. Area representation of the performance in terms of area-based segmentation assessment metrics [4]

Dice Similarity Coefficient (DSC) is a common similarity metric for representing the percentage or amount of area common to the assessed delineation $A$ and the reference delineation $M$. Its value range between 0 (no overlap) and 1 (perfect agreement) [4]. The DSC metric is expressed by formula:

$$
D S C=\frac{2|A \cap M|}{|A|+|M|}=\frac{2 . T P}{2 . T P+F P+F N}
$$

where $\mathrm{C}$ is the intersection operator and $|\cdot|$ represents the number of pixels in the corresponding pixel set.

True-Positive Ratio (TPR) also known as sensitivity, corresponds to the amount of properly labeled pixels as lesion with respect to the amount of lesion pixels from the reference delineation [4]. The TPR metrics is expressed by formula:

$$
T P R=\frac{|A \cap M|}{|M|}=\frac{T P}{T P+F P}
$$

True-Negative Ratio (TNR) also known as specificity, corresponds to the amount of background correctly labeled. The TNR metrics is expressed by formula:

$$
T N R=\frac{|\bar{A} \cap \bar{M}|}{|M|}=\frac{T N}{T N+F P}
$$

Accuracy (ACC) quantifies the amount of properly labeled pixels as lesion with respect to the amount pixels both of assessed delineation $A$ and delineation $M$. The ACC metrics is expressed by formula:

$$
A C C=\frac{T P+T N}{T P+T N+F P+F N}
$$

\section{RESULTS AND ANALYSIS}

This section describes the results of the implementation of the method proposed. The results of implementation consist of the segmentation results using the level set algorithm that combined with morphological operations and the validation results using area-based segmentation assessment metrics. 


\subsection{Segmentation}

The test image used in this research comes from our breast ultrasound image database and the lesion's contour is manually delineated by radiologists. This delineated area, denominated Ground Truth (GT) that used on validation process to evaluate the accuracy of segmentation methods proposed. Two of 30 breast ultrasound images used in our research can be seen in Table 1.

In Table 1 it can be seen two examples of ultrasound images that indicated there are the breast tumor. On the left column contain the ultrasound images with gray-scale image type, where the bubble black is a breast tumor. The images on the right column are the results of the breast tumor segmentation of the left images who done manually by the radiologists. The results of this manual segmentation are used as a comparison to the results of the segmentation of methods proposed in this study.

The segmentation process in this research using three methods based on level set that each method combined with morphological operations. Three level set models that compared in this paper are the C-V model, the SBGFRLS model and the DRLSE model. Then the use of morphological operations in the three level set models aimed to obtain more accurate segmentation results. The examples of segmentation results of the three methods that proposed in this paper can be seen in Table 2.

Table 1. Two samples of Breast Ultrasound Images in Data set

Original image

Table 2. Segmentation Results

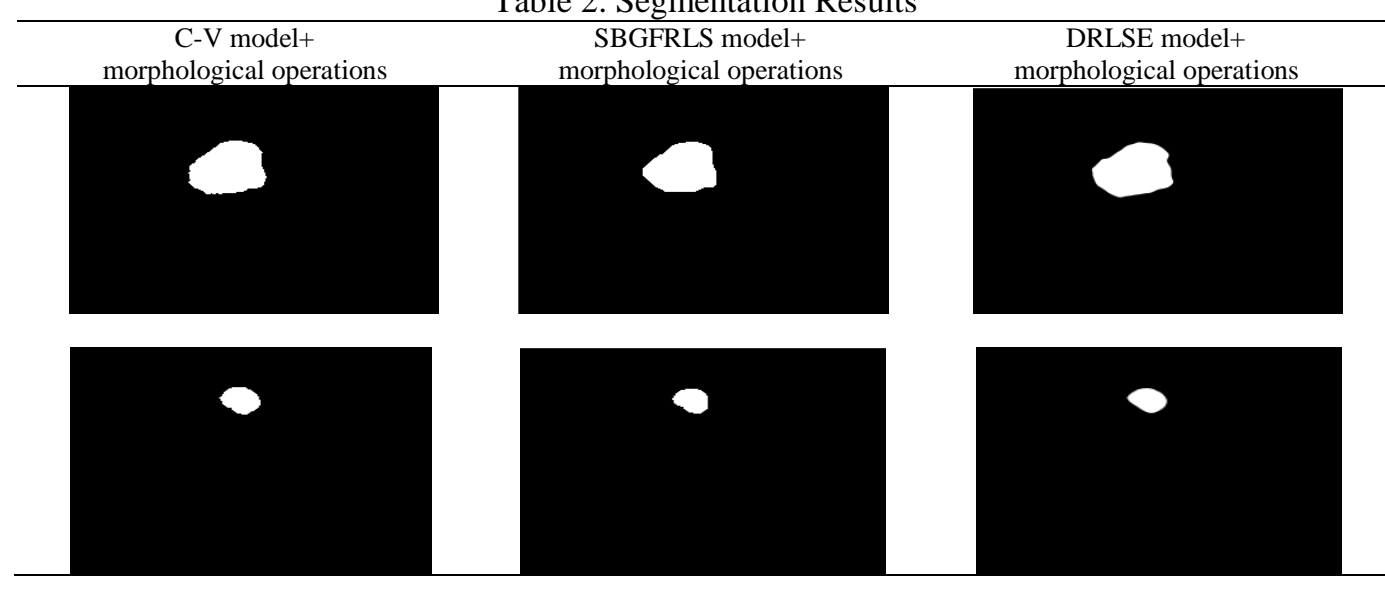

We can see in Table 2 that the results of this segmentation process in the form of binary image that divided into two, namely a white area is the breast lesion and black area is the background. This binary image is called the Region of Interest (ROI) image. In Table 2 it also can be seen that the results of the three methods proposed are significantly quite different. Then on the images of the segmentation results were done the validation process to see which method is more accurate.

\subsection{Validation}

Furthermore, the results of the segmentation process needs to be performed a validation process to ensure the accuracy of the segmentation method proposed. The validation process is done by comparing the 
ROI image with the GT image by using area-based segmentation assessment metrics. In this validation process has been generated the value of similarity between the ROI image with the GT image. The summary results of the validation value from the 30 test images in this research can be seen in Table 3 .

Based on the results obtained in Table 3, we can summarize that the combination of the C-V model and morphological operations has a very good performance in term of segmentation breast lesion with Dice Similarity Coefficient (DSC) is $94 \%$ and True-Positive Ratio (TPR) is 94\%, give the best than the others. However True-Negative Ratio (TNR) is worse than DRLSE model that combined with morphological operations and accuracy has a same with DRLSE model that combined with morphological operations.

Table 3. Evaluation Metrics

\begin{tabular}{|c|c|c|c|c|}
\hline Methods & $\begin{array}{c}\text { Mean DSC } \\
(\%)\end{array}$ & $\begin{array}{c}\text { Mean TPR } \\
(\%)\end{array}$ & $\begin{array}{c}\text { Mean TNR } \\
(\%)\end{array}$ & $\begin{array}{c}\text { Mean ACC } \\
(\%)\end{array}$ \\
\hline C-V model+morphological operations & 94 & 94 & 99 & 94 \\
\hline SBGFRLS model+morphological operations & 90 & 86 & 99 & 93 \\
\hline DRLSE model+morphological operations & 90 & 87 & 100 & 94 \\
\hline
\end{tabular}

\section{CONCLUSION}

In this paper, we have presented a comprehensive comparison of three level set methods that combined with morphological operations for segmentation of breast ultrasound images. Three level set models used in this paper are the Chan Vese $(\mathrm{C}-\mathrm{V})$ model, the Selective Binary and Gaussian Filtering Regularized Level Set (SBGFRLS) model and the Distance Regularized Level Set Evolution (DRLSE) model. The performance evaluation of our methods were been demonstrated by the experimental results with used 30 breast ultrasound images, which the lesion had been delineated manually by radiologists. The performance of $\mathrm{C}-\mathrm{V}$ model that combined with morphological operations was significantly better than the other two methods. It was indicated from the average value of precision, which is $94 \%$ for Dice Similarity Coefficient (DSC) metric that much higher than the SBGFRLS model that combined with morphological operations (90\%) and the DRLSE model that combined with morphological operations (90\%).

\section{REFERENCES}

[1] A. Jemal, et al., "Global Cancer Statistics", CA: A Cancer Journal for Clinicians, vol. 61, pp. 69-90, Mar 2011.

[2] L. Gao, et al., "Phase- and GVF-Based Level Set Segmentation of Ultrasonic Breast Tumors", Journal of Applied Mathematics, vol. 2012, 2012.

[3] H.D. Cheng, et al., "Automated Breast Cancer Detection and Classification Using Ultrasound Images: A Survey", Pattern Recognition, vol. 43, pp. 299-317, 2010.

[4] D.P. Lestari, et al., "A Segmentation Algorithm for Breast Lesion Based on Active Contour Model and Morphological Operations", Advanced Science Engineering and Medicine, vol. 7, pp. 920-924, Oct 2015.

[5] H. Kumar, et al., "Segmentation and Feature Extraction of Ultrasound Images by Modified Level Set Method and Chain-Vese Methods Using SRAD Filter", International Journal of Advanced Research in Computer and Communication Engineering, vol. 1, pp. 171-177, May 2012.

[6] G. Zhu, et al., "Boundary-Based Image Segmentation Using Binary Level Set Method", Optical Engineering, vol. 46, no. 5, May 2007.

[7] K. Zhang, et al., "Active Contours with Selective Local or Global Segmentation: A New Formulation and Level Set Method", Image and Vision Computing, vol. 28, pp. 668-676, Apr 2010.

[8] C. Xu, et al., Handbook of Medical Imaging Volume 2: Medical Image Processing and Analysis. Bellingham, USA: SPIE Press, 2000.

[9] X. Chang, et al., "A Study of Image Segmentation Based on Level Set Method", in International Conference on Advanced Computer Science and Electronics Information, pp. 360-363, 2013.

[10] C. Li, et al., "Distance Regularized Level Set Evolution and Its Application to Image Segmentation", IEEE Transactions on Image Processing, vol. 19, pp. 3243-3254, Dec 2010.

[11] T.F. Chan and L.A. Vese, "Active Contour without Edges", IEEE Transactions on Image Processing, vol. 10, pp. 266-277, Feb 2001.

[12] R.C. Gonzalez and R.E. Woods, Digital Image Processing. Singapore: Pearson Prentice Hall, 2002.

[13] K.O. Babalola, et al., "Comparison and Evaluation of Segmentation Techniques for Subcortical Structures in Brain MRI", in MICCAI 2008 Part I LNCS, vol. 5241, pp. 409-416, 2008.

[14] K. Saini, et al., "Level Set Based on New Signed Pressure Force Function for Echocardiographic Image", International Journal of Innovation and Applied Studies, vol. 3, pp. 560-569, June 2013.

[15] M. Goyal, "Morphological Image Processing", International Journal of Computer Science and Technology, vol. 2, pp. 161-165, Dec 2011. 


\section{BIOGRAPHIES OF AUTHORS}
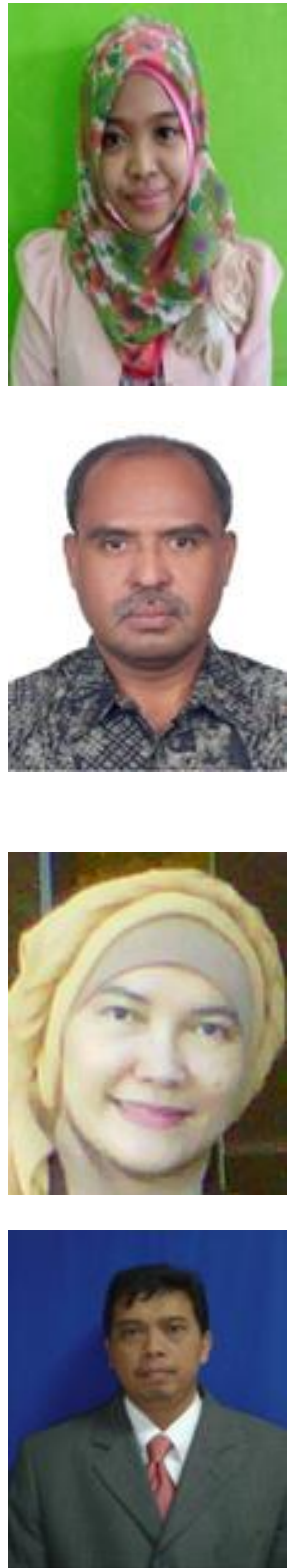

Prof. Sarifuddin Madenda - Currently Director of High School of Computer Science and Management Jakarta (STMIK Jakarta STI\&K). He received the B.S. degree from University of Indonesia, in 1989, the M.S. degree from Institute National des Sciences Appliquées de Lyon (INSA de Lyon) - French, in 1992, and the Ph.D. degree from University of Burgundy - French, in 1995. From 1995 to 1996, he was a Research Associate at LIESIB Laboratory - University of Burgundy. In 1997, he became a Lecturer in Computer Science Department, Gunadarma University - Indonesia. From 2002 to 2007, he was a researcher at Academic Research Consortium on digital imaging, video, audio and multimedia (CoRIMedia), Canada. His research interests are image processing: image compression, color imaging, image database and searching, medical image analysis, implementing of image processing algorithms on FPGA for real time image analysis. Email: sarif@staff.gunadarma.ac.id

Dr. Ernastuti - has received B.S. degree in Mathematics from University of Indonesia in December 1985, and the M.S. in Computer Science from University of Indonesia, in July 1994, and $\mathrm{PhD}$ degrees in Computer Science from Gunadarma University, Indonesia, in April 2008. She is currently an associate professor in the faculty of computer science and Information Engineering, Gunadarma University. Her current research interest include graph theory and combinatorial optimization, graph-theoretic interconnection networks, parallel and distributed computing, and design and analysis of algorithms. Email: ernas_tuti@yahoo.co.id

Eri Prasetyo Wibowo born in Kendal, Indonesia, in 1966. He received the B.S degree in Electronics and Instrumentation from Gadjah Mada University, Indonesia, in 1992, the M.S. degree in Information System from Gunadarma University, Indonesia, in 1994 and The Ph.D. degree in Electronics Informatics from the Burgundy University, France, in 2005. He is member of IEEE, ACM, Professional Organization in the field of Information and Computer Technology (IPKIN), APTIKOM . He has been worked as Secretary of Doctoral Program in Information Technology at Gunadarma University. He was member of EACOVIROE Project to promoted Master VIBOT which founded by Erasmus Mundus. His current interests are in Real time Image processing applications and System On CHIPs Design. Email: eri@staff.gunadarma.ac.id 